\title{
Manipulation of lymphoid microenvironments in nonhuman primates by an inhibitor of the lymphotoxin pathway
}

\author{
Jennifer L. Gommerman, ${ }^{1}$ Fabienne Mackay, ${ }^{2}$ Elina Donskoy, ${ }^{3}$ Werner Meier, ${ }^{4}$ \\ Pauline Martin, ${ }^{2}$ and Jeffrey L. Browning ${ }^{1}$ \\ ${ }^{1}$ Department of Exploratory Sciences, \\ ${ }^{2}$ Department of Pre-clinical and Clinical Development Sciences, \\ ${ }^{3}$ Department of Bioassay, and \\ ${ }^{4}$ Department of Drug Discovery, Biogen Inc., Cambridge, Massachusetts, USA
}

\begin{abstract}
Reticular networks in lymphoid organs play critical roles in the organization of local microenvironments. A number of these elements are maintained by continual signaling through the lymphotoxin system. Evaluation of the lymphotoxin (LT) pathway in primates using a fusion protein decoy provides a unique opportunity to assess modulation of splenic microenvironments in a species with considerably greater background immunological activity compared with rodents. Within the germinal center microenvironment, treatment resulted in a collapse of follicular dendritic cell (FDC) networks and in the disappearance of a ringlike network of immune complex-carrying cells, although some other attributes of the germinal center appeared to be unaltered. Treatment also resulted in changes in the splenic marginal zone, a microenvironment where the architecture is notably different from that of the rodent. Cessation of treatment and recovery allowed us to monitor reemergence of these cell types and revealed that FDCs rely on LT-dependent signals to recompact into appropriately positioned tight networks. Despite the loss of FDC networks, the primary $\mathrm{Ab}$ response to keyhole limpet hemocyanin was unaltered over a 20-day period. Manipulation of these microenvironments may represent a novel approach to modulating immune function in human disease.
\end{abstract}

J. Clin. Invest. 110:1359-1369 (2002). doi:10.1172/JCI200215975.

\section{Introduction}

The lymphotoxin (LT) system is critically involved in the organization of lymphoid microenvironments, and some of this control over cellular positioning stems from effects on chemokine secretion by the stromal elements and the regulation of surface adhesion molecules (1). A heteromeric $L T \alpha / \beta$ ligand expressed on a subset of follicular resting B cells and activated B, T, and NK cells interacts with the lymphotoxin- $\beta$ receptor $(\mathrm{LT} \beta \mathrm{R})(2,3)$. Interruption of LT $\beta \mathrm{R}$ signaling either by

Received for publication May 21, 2002, and accepted in revised form August 27, 2002.

Address correspondence to: Jeffrey L. Browning, Biogen Inc., 12 Cambridge Center, Cambridge, Massachusetts 02142, USA. Phone: (617) 679-3312; Fax: (617) 679-2304;

E-mail: Jeff_Browning@biogen.com.

Fabienne Mackay's present address is: Garvan Institute of Medical Research, St. Vincent's Hospital, Darlinghurst, Australia. Conflict of interest: The authors are full-time employees of Biogen Inc. and participate in its stock option program.

Nonstandard abbreviations used: lymphotoxin (LT); lymphotoxin- $\beta$ receptor (LT $\beta R$ ); follicular dendritic cell (FDC); marginal zone (MZ); lymph node (LN); immune complex (IC); germinal center (GC); transmissible spongiform encephalopathy (TSE); keyhole limpet hemocyanin (KLH), peroxidase-antiperoxidase (PAP); alkaline phosphatase (AP); horseradishperoxidase (HRP); nitro blue tetrazolium/5-bromo-4-chloro-3indolyl phosphate (NBT/BCIP); smooth muscle actin (Asm-1); tyramide signal amplification (TSA); incomplete Freund's adjuvant (IFA); inner-MZ (i-MZ); nitrophenyl (NP); outer-MZ (o-MZ). genetic disruption or pharmacologically with Ab's or a receptor-Ig decoy, results in the collapse of several microenvironments. These microenvironments include a CXCL13 chemokine-secreting reticular stromal network in the follicle, the MAdCAM-1-positive endothelial-like cells lining the marginal sinus, and the follicular dendritic cell (FDC) network (4-6). The marginal zone (MZ) is also disrupted in the rodent spleen, and this microenvironment contains a unique type of $\mathrm{B}$ cell that is activated in a T-independent fashion. This microenvironment has been implicated recently as a potential source of autoreactive $B$ cells $(7,8)$.

FDC networks dominate the $\mathrm{B}$ cell regions of the spleen, lymph nodes (LNs), and Peyer's and colonic patches and appear ectopically in organized lymphoid structures in chronically inflamed pathological settings (9-11). FDCs trap immune complexes (ICs) on the surface of their dendrites either by complement receptors CD35 (CR1) or Fc receptors (12). These ICs are thought to play a role in the affinity maturation of humoral responses within the germinal center (GC) reaction (13), and this antigen trapping greatly prolongs the half-life of antigen in an organism following immunization (14). Inhibition of the LT axis led to the rapid disappearance of FDC networks in the rodent, which was surprising given their ability to retain antigen for long periods of time and apparent stability (6). Work with $L T \alpha, L T \beta$, and $L T \beta R$ knockout mice, as well 
as lymphocyte reconstitution experiments, support the concept that LT is critical for FDC formation $(15,16)$. FDCs exist in several forms in the rodent representing distinct stages in mesenchymal cell differentiation. For example, FDC precursors have been demonstrated within the lymphoid tissues (17). In addition, two mature forms of FDC can be distinguished morphologically, with one type residing in the primary follicle, also called the mantle in primate tissues, and the other type existing in the secondary follicle or GC (9).

There are a number of clinically relevant reasons to understand the biology of FDCs as well as the maintenance of reticular networks that form splenic microenvironments such as the MZ. First, in general, manipulation of only some components of the immune system is desirable to dampen activity in autoimmune disease, and as such, FDCs represent a relatively selective therapeutic target. Second, the normal survival signals that affect GC B cells may also be critical for survival of some forms of LN-residing lymphomas (18). Third, the ability to trap IC on the surface of FDCs for prolonged periods of time may be exploited by pathogens. For example, FDCs serve as a reservoir for HIV virions (19, 20), and infectious HIV particles have been shown to remain on FDC networks following antiretroviral treatment (21). Likewise, in animal models of transmissible spongiform encephalopathies (TSEs), replication of the proteinacious infectious pathogen $\operatorname{PrPsc}$ occurs specifically in FDCs (22), and a single pretreatment with LT $\beta$ R-Ig to eliminate FDCs results in a delay of disease onset (23). Fourth, FDCs may provide an interesting window into the spectrum of fibroblastoid cell types. For example, the hyperplastic synovial fibroblasts in rheumatoid arthritis take on FDC-like characteristics and this transition may be important in the pathological process (24). Lastly, the MZ is an additional microcompartment that may contain autoreactive $B$ cells and play a role in the progression of autoimmune disease $(7,8)$. Therefore, manipulation of this specialized compartment may be useful in the treatment of autoimmune diseases such as systemic lupus erythematosus.

Since LT $\beta$ R-Ig may serve as a potential therapeutic agent in humans, and given the scarcity of human/primate histological information on in vivo IC trapping, FDC dissolution/recovery, and MZ architecture, we performed a study in cynomolgus monkeys testing the effects of LT $\beta$ R-Ig treatment. To this end, we have asked three questions: First, does LT $\beta R$-Ig affect FDC networks and the splenic MZ in primates where, compared with rodents, the splenic architecture is notably different and there is considerably greater baseline immunological activity? Second, given the importance of IC retention in maintaining a long-term reservoir of both innocuous antigen as well as pathogens in the case of HIV or PrPSc, does LT $\beta$ R-Ig treatment impact FDC function as measured by IC deposition in primates, and does it impair Ig responses to an antigen? Finally, do FDC networks have the capacity to recover from LT $\beta$ R-Ig treatment, and if so, has their architec- ture been altered? To address these questions, we quantified FDCs and their ability to capture IC, analyzed the splenic $\mathrm{MZ}$, and assessed the $\mathrm{Ab}$ response to keyhole limpet hemocyanin (KLH) following a 4-week repeat dose study in which cynomolgus monkeys were treated with a human LT $\beta$ R-Ig fusion protein.

\section{Methods}

Animals. Cynomolgus monkeys (Macaca fascicularis) were used in all studies. Forty-six animals (23 males and 23 females) were selected with ages ranging from 3 to 7 years and weighing $2.5-4 \mathrm{~kg}$. These animals were segregated into five groups: vehicle control (saline injection), those intravenously injected with 0.8 $\mathrm{mg} / \mathrm{kg}, 8 \mathrm{mg} / \mathrm{kg}$, and $40 \mathrm{mg} / \mathrm{kg} \mathrm{LT} \beta \mathrm{R}-\mathrm{Ig}$, and those subcutaneously injected with $8 \mathrm{mg} / \mathrm{kg}$ LT $\beta$ R-Ig. Injections were performed once a week on days $1,8,15$, and 22 , and then animals were sacrificed on day 29 (for nonrecovery animals), day 85 (for 8-week recovery), or day 113 (for 12-week recovery). The human LT $\beta R$ human $I_{g G_{1}} \mathrm{Fc}$ domain construct was described previously (25). Some animals from each group were selected for a $25-\mathrm{mg} / \mathrm{kg}$ intravenous injection of endotoxin-free goat peroxidase-antiperoxidase (PAP; ICN Radiochemicals Inc., Costa Mesa, California, USA) 24 hours before sacrifice. High concentrations of PAP were required because IC complexes are rapidly cleared to the liver $(26,27)$. All animals were housed at Sierra Biomedical Inc. (Sparks, Nevada, USA), as specified in the United States Department of Agriculture Animal Welfare Act and as described in the Guide for Care and Use of Laboratory Animals.

Immunohistochemistry. Spleens and mesenteric LNs were suspended in OCT (Sakura Finetek, Torrance, California, USA), frozen and sectioned at $5 \mu \mathrm{m}$, fixed with acetone, and then stained according to the protocol as described (4). Briefly, nonspecific binding was blocked using TBS $/ 5 \%$ BSA $/ 5 \%$ horse serum $/ 0.05 \%$ Tween 20 , and the sections were stained with Ber-Mac-DRC (antiCD35) (DAKO Corp., Carpinteria, California, USA), or FDC-specific Ab's (clone CNA.42, unknown FDC-specific antigen), or R42/3 (anti-CD21), as well as antiCD20 and anti-CD3 Ab's (all DAKO Corp.). Anti-mouse IgG alkaline phosphatase (AP; Southern Biotechnology Associates, Birmingham, Alabama, USA), anti-mouse IgG horseradish-peroxidase (HRP), or anti-mouse IgM HRP (both Southern Biotechnology Associates) were used as secondary reagents, and control slides were stained with secondary reagents alone (data not shown). AP labeling was followed by nitro blue tetrazolium/5bromo-4-chloro-3-indolyl phosphate (NBT/BCIP) red or blue color (Vector Laboratories, Burlingame, California, USA), and HRP labeling was followed by 3,3'diaminobenzidine development (Vector Laboratories). Some slides were counterstained with Giemsa. For fluorescent detection, spleen sections were stained with anti-IgD FITC, Ber-Mac-DRC (anti-CD35), L26 (antiCD20), Ki-67 (all from DAKO Corp.), Asm-1 (antismooth muscle actin; PROGEN Biotechnik GmbH, 
Heidelberg, Germany), biotinylated anti-VCAM-1 (PharMingen, San Diego, California, USA), and tyramide signal amplification-rhodamine (TSA-rhodamine) (NEN Life Science Products Inc., Boston, Massachusetts, USA) to detect PAP-associated peroxidase. Animals that did not receive PAP injections exhibited some TSA-rhodamine signal in the red pulp due to endogenous peroxidase in macrophages but not in the follicle (data not shown). Alexa fluors 568- and 680-conjugated anti-mouse IgG and streptavidin-conjugated alexa fluor 568 and 488 were used as secondary reagents (Molecular Probes Inc., Eugene, Oregon, USA). Stained sections were then visualized on a Leica scanning confocal microscope or Leica fluorescent microscope.

In vitro IC deposition assay. Goat PAP at $1 \mathrm{mg} / \mathrm{ml}$ was preincubated with $10 \%$ fresh human serum for $30 \mathrm{~min}$ utes and then applied to nonacetone-fixed frozen spleen sections for 1 hour at room temperature. Slides were then stained with anti-CD20 Ab's followed by FITC-conjugated anti-mouse IgG stain and TSA-rhodamine development. No PAP deposition was detected in the absence of goat PAP injection (data not shown). Morphometric analysis of spleen and LN sections. Spleen and $\mathrm{LN}$ sections were imaged at $\times 16$ and imported into Openlab software (Improvision, Coventry, England). Only slides stained by conventional nonfluorescent immunohistochemical methods were subjected to this analysis. Using a tool to discriminate based on shade differences, FDC network areas were calculated as well as total tissue areas. Settings for the shading tool were kept constant for all sections. Because of the interanimal variability, this approach allowed us to quantify the average affect of treatment on FDC networks throughout the cohort. In the case of PAP quantification, PAP networks located in white pulp areas were enumerated and represented as number of PAP networks per square millimeter of tissue. Typically, three overlapping fields of view (3-4 $\mathrm{mm}^{2}$ each) per slide were compiled to assess both FDC areas and PAP networks. All statistical calculations were generated using the Student $t$ test. The methods defined here are similar to other analyses of FDC content performed with human samples $(28,29)$.
Evaluation of anti-KLH responses. One hundred micrograms KLH (Sigma-Aldrich, St. Louis, Missouri, USA) was prepared as a 1:1 emulsion with incomplete Freund's adjuvant (IFA) and administered by intradermal injection to the lateral abdominal region at day 8 subsequent to the second dose of LT $\beta R$-Ig. Serum was collected for determination of anti-KLH Ab's prior to KLH dosing, and on days 15, 22, and 28 (i.e., days 7, 14, and 20 after immunization). Titers were then determined by the ELISA method. Briefly, plates were coated with KLH, then blocked to minimize nonspecific binding. Subsequently, standards and serum were added to the plate followed by anti-monkey IgG Ab conjugated to HRP and HRP development (Cappel/ICN Pharmaceuticals Inc., Costa Mesa, California, USA). Titers were normalized over preimmunized anti-KLH levels for each animal.

\section{Results}

CD35 staining of FDC networks in spleen and LNs is eliminated following treatment with LT $\beta R$-Ig. LT $\beta \mathrm{R}-\mathrm{Ig}$ treatment in mice resulted in the disappearance within several days of FDC markers in the spleen (6). Primates have significantly greater baseline immunological activity, and the basic architecture of the primate spleen differs from that in the rodent. For example, unlike the rodent spleen, the $\mathrm{T}$ cell-rich periarteriolar lymphoid sheath in monkeys is small and tangential to the predominant follicles, almost all of which contain a GC. Because of these variables, it was uncertain whether the human LT $\beta$ R-Ig would be an effective modulator of FDC status and function in primates. Therefore, cynomolgus monkeys were given weekly injections of LT $\beta$ R-Ig for 4 weeks, followed by an assessment of CD35 expression on FDC networks. The LT $\beta$ R-Ig construct was well tolerated in cynomolgus monkeys, and $\mathrm{Ab}$ responses to this therapeutic agent were barely detectable over the course of the study (data not shown). Splenic sections from control animals reveal CD35-positive cells (red) as a tight network within the white pulp areas counterstained with Giemsa blue (Figure 1, $a$ and $b$ ). The CD35 staining protocol

\footnotetext{
Figure 1

FDC networks in the spleen are eliminated in animals treated with LT $\beta \mathrm{R}-\mathrm{lg}$. Representative spleens from untreated ( $\mathbf{a}$ and $\mathbf{b})$, treated ( $8 \mathrm{mg} / \mathrm{kg} \mathrm{LT} \beta \mathrm{R}-\mathrm{lg}$ ) (c and $\mathbf{d}$ ), and recovery animals $(8 \mathrm{mg} / \mathrm{kg}$ treatment followed by 3-month recovery) (e and $\mathbf{f}$ ) were stained with anti-CD35 Ab's and developed with AP-red. Spleens were counterstained with Giemsa and photographed at $\times 25(\mathbf{a}, \mathbf{c}, \mathbf{e})$ or $\times 100(\mathbf{b}, \mathbf{d}, \mathbf{f})$.
}
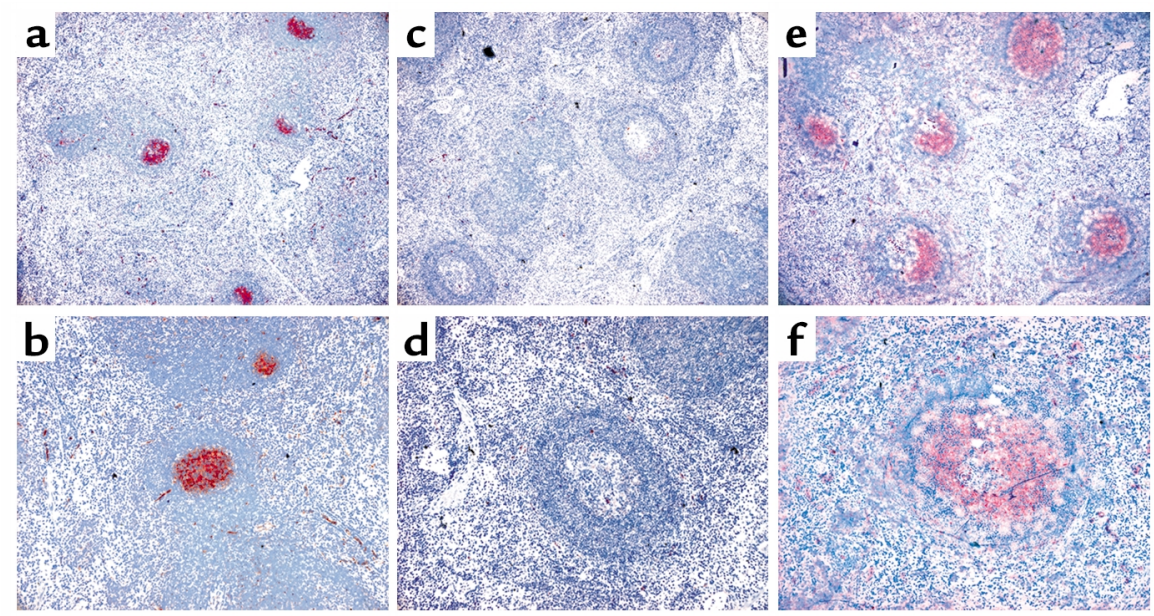

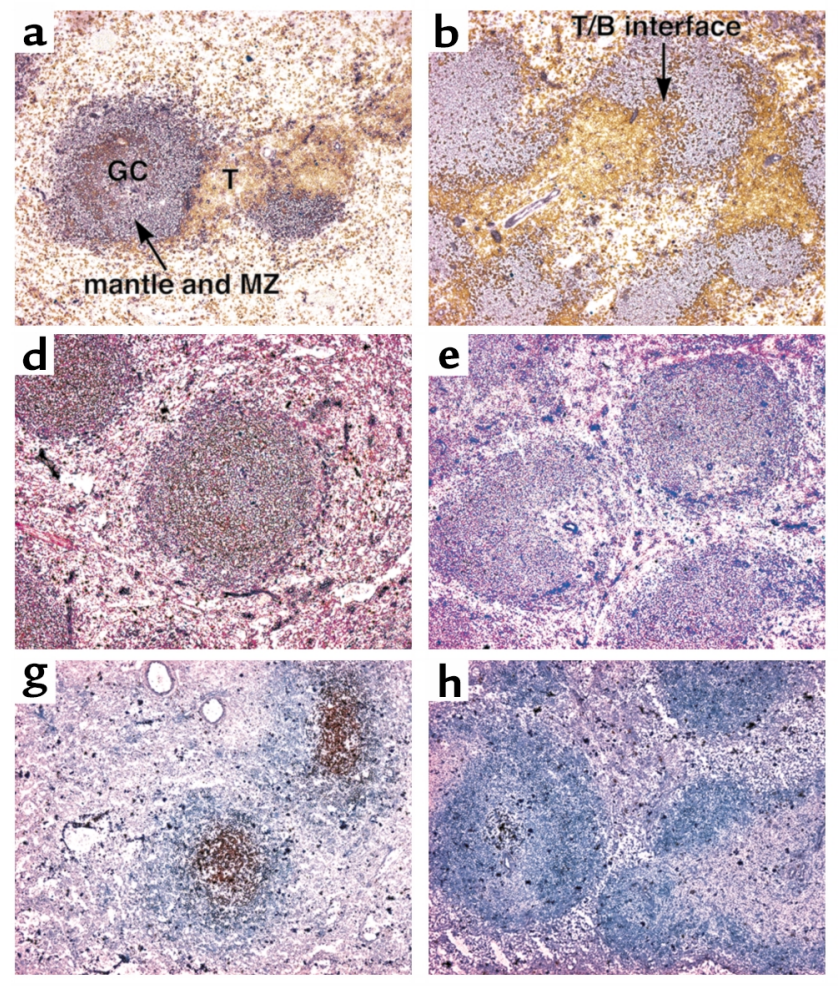

was adjusted to detect only CD35-bright cells (FDCs) and not CD35-dim B cells in the mantle or marginal zones. In contrast to control splenic white pulp, the white pulp areas in the LT $\beta$ R-Ig-treated animals contained vastly reduced numbers of FDC networks (Figure $1, \mathrm{c}$ and d). Similar data were obtained using two other FDC-specific markers, specifically the CNA.42 clone, which stains for an unknown FDC-specific nonlineage marker (Figure 2, d-f), as well as the CD21-specific clone R42/3 (Figure 2, g-i) (30). B cell and T cell areas were also examined using $\mathrm{CD} 20$ and $\mathrm{CD} 3$ stains, respectively (Figure 2, a-c). No obvious changes in $\mathrm{B}$ cell or $\mathrm{T}$ cell areas were noted (see identification of white pulp area compartments in a spleen from an untreated animal: note the presence of T cells in what is likely a GC; Figure 2a). However, consistent with what is observed in the rodent (4), some blurring of the T cell/B cell interface was observed with treatment. LT $\beta \mathrm{R}-\mathrm{Ig}$ treatment of mice leads to an increase in both $\mathrm{T}$ and $\mathrm{B}$ cell counts in the blood 1-2 weeks after treatment, and the reasons for this leukocytosis are unknown (unpublished observations). Accordingly, in the peripheral blood of treated monkeys, $\mathrm{CD} 4^{+} \mathrm{T}$ cell, $\mathrm{CD} 8^{+} \mathrm{T}$ cell, and $\mathrm{CD} 20^{+} \mathrm{T}$ cell numbers were found to increase slightly in a dose-dependent manner; however, these changes were small relative to the mouse, and there was considerable interanimal variability (data not shown).

A closer examination of FDC networks by confocal microscopy was performed to analyze the composition of the primate splenic GCs. In the splenic white pulp, IgD-negative GCs were observed surrounded by an IgD-positive mantle zone (green) (Figure 3a). Within the IgD-negative GC, CD35-positive FDCs (red) were

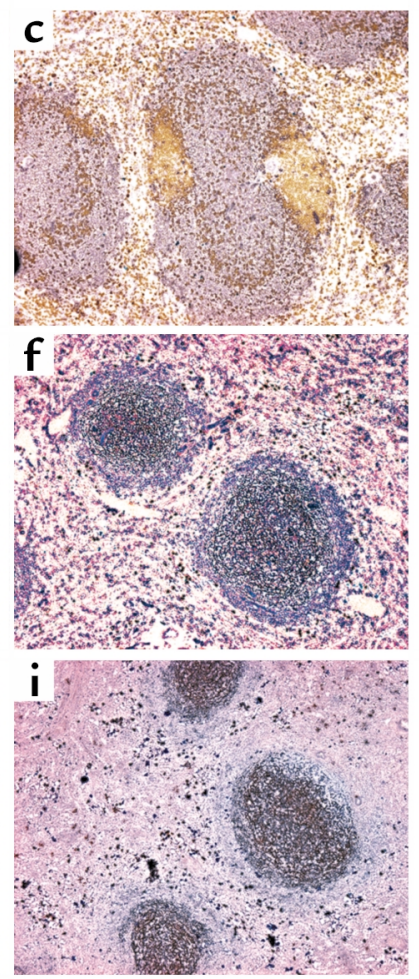

\section{Figure 2}

$B$ cell and T cell areas are mostly unaltered by LT $\beta R$-Ig treatment, but CD21 staining is diminished. Representative spleens from untreated (a, d, $\mathbf{g})$, treated (8 $\mathrm{mg} / \mathrm{kg}$ LT $\beta$ R-Ig) (b, e, h), and recovery animals $(8 \mathrm{mg} / \mathrm{kg}$ treatment followed by 3-month recovery) $(\mathbf{c}, \mathbf{f}, \mathbf{i})$ were stained with anti-CD20 Ab's (blue). Sections were costained with anti-CD3 Ab's (brown) (a-c), or the FDCspecific clone CNA.42 (brown) (d-f), or the CD21-specific R42/3 clone (dark brown) (g-i). Spleens were photographed at $\times 100$. T, T cell zone; $\mathrm{T} / \mathrm{B}$ interface, $\mathrm{T}$ cell/B cell interface. observed in a tight network, intermingling with Ki67-positive cells (blue), indicating the presence of proliferating cells within the GC (31). When spleen sections from LT $\beta R$-Ig-treated animals were examined, the CD35 staining was virtually gone from the follicle (Figure 3b). However, the remains of a GC were apparent as evidenced by the IgD-negative area. The amount of Ki-67 staining was approximately the same between treated and nontreated samples, and this was determined by examining Ki-67 staining in isolation on the confocal microscope (data not shown).

Morphometric analysis was used to quantify the reduction in FDC networks where CD35-positive areas were measured and the data are presented as a percentage of the total tissue area. It was important to quantify the affect on CD35-positive FDC networks because there was considerable interanimal variability. The percentage of splenic area occupied by FDC networks was significantly reduced at the $0.8 \mathrm{mg} / \mathrm{kg}, 8 \mathrm{mg} / \mathrm{kg}$, and $40 \mathrm{mg} / \mathrm{kg}$ doses $(P<0.002,0.003$, and 0.003 , respectively) (Figure 4a). Similar reductions were observed in the $\mathrm{LN}$ at the $0.8 \mathrm{mg} / \mathrm{kg}$, $8 \mathrm{mg} / \mathrm{kg}$, and $40 \mathrm{mg} / \mathrm{kg}$ doses $(P<0.03,0.04$, and 0.02 , respectively) (Figure $4 \mathrm{~b}$ ). We have found that FDC networks in murine LNs are also reduced with treatment (ref. 6 and unpublished observations), and, moreover, the occasional residual mesenteric $\mathrm{LNs}$ in $\mathrm{LT} \alpha^{-/-}$mice are similarly devoid of FDCs (32). In addition, the number of FDC networks per square millimeter of tissue was correspondingly reduced with treatment (data not shown). Hence, although these animals exhibit significant preexisting GC activity, CD35 staining of FDC networks in both the spleen and the $\mathrm{LN}$ is dramatically reduced with LT $\beta$ R-Ig treatment at doses as low as $0.8 \mathrm{mg} / \mathrm{kg}$. 

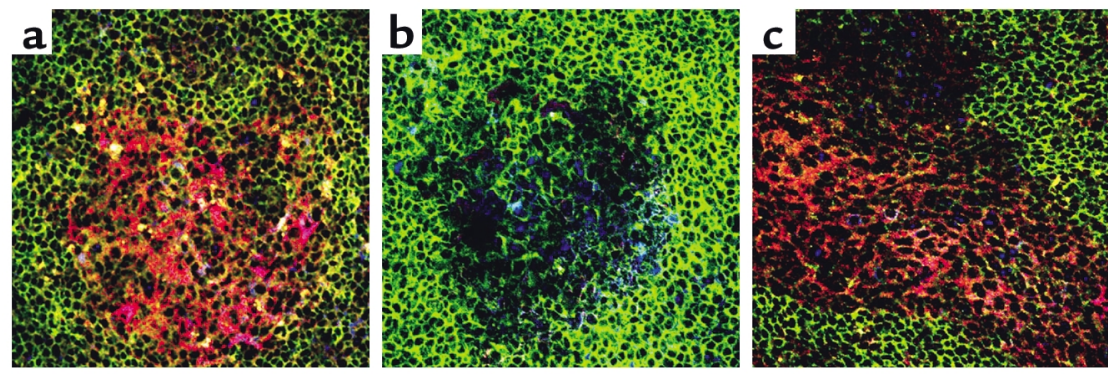

\section{Figure 3}

Confocal analysis of splenic GCs. Spleens from untreated (a), treated ( $8 \mathrm{mg} / \mathrm{kg}$ LT $\beta R-\lg )(\mathbf{b})$, and recovery animals $(8 \mathrm{mg} / \mathrm{kg}$ treatment followed by 3 -month recovery) (c) were stained with anti-IgD (green), anti-CD35 (red), and Ki67 (blue). Spleens were photographed under oil immersion at $\times 400$ on a scanning confocal microscope. Ki-67/CD35 double positive cells appear as magenta colored in (a).

Collapsed FDC networks can recover. Mouse studies suggest that FDC networks require approximately 1-2 weeks to form (33). In preliminary studies, examination of spleens from primates dosed with a single injection of $3-5 \mathrm{mg} / \mathrm{kg}$, recovery occurred within a 3- to 5-week window (data not shown). In the major study with repeat dosing, recovery was not detected at 2 months after cessation of treatment ( $8-40 \mathrm{mg} / \mathrm{kg}$ dose groups), but did reappear at 3 months, and the two animals exhibiting the least recovery were from the $40-\mathrm{mg} / \mathrm{kg}$ group (Figure 4c). From examination of the serum levels of LT $\beta R-I g$ in these studies, we speculate that levels need to fall to at least less than $1-5 \mu \mathrm{g} / \mathrm{ml}$ before FDCs begin to reemerge (data not shown), and this is consistent with a 2- to 3 -week recovery period once LT $\beta$ R-Ig has been sufficiently cleared. In many of the animals that were allowed to recover, the splenic CD35-positive FDC networks appeared larger and more diffuse in comparison with FDC networks from vehicle-treated animals (Figure 1, b vs. f, and Figure 3, a vs. c). We postulate that when FDC networks re-form, it takes time for them to coalesce into a tight, compact structure within the GC. Therefore, treatment with LT $\beta$ R-Ig does not result in permanent disruption of FDC networks in immunologically active adult primates. When mesenteric LNs were examined, treatment with LT $\beta \mathrm{R}$ Ig results in a similar decrease in FDC networks. However, unlike the spleen (Figure 4c), FDC networks in the $\mathrm{LN}$ were slow to recover, and the 3-month recovery animals were not statistically different from treated animals (data not shown).

The MZ of the spleen is altered with LT $\beta R$-Ig treatment. Studies in the rodent demonstrate that a number of MZ markers are lost upon LT $\beta$ R-Ig treatment (4). Unlike the rodent, the human MZ appears to lack a $M Z$ sinus, and the $M Z$ is divided into an inner and outer compartment separated by a layer of myofibroblasts (34). This myofibroblast layer can be visualized with a mAb to smooth muscle actin (Asm-1) (Figure 5, $\mathrm{a}-\mathrm{f}$; red), and this reagent also stains arterioles (see staining within the GC, Figure 5d). Adjacent to this layer on the white pulp side is the IgD-negative/low inner-MZ (i-MZ) (Figure 5a, arrows), which borders the $\operatorname{IgD}$ positive (green) mantle zone. Spleens from animals treated with LT $\beta$ R-Ig show no evidence of alteration of the Asm-1-positive layer; however, upon close inspection of this region, the IgD-positive mantle was found to merge into the Asm-1-positive layer without interruption by an IgD-negative/low i-MZ (Figure $5 \mathrm{~b}$ ). In the mouse, VCAM-1 is also expressed in the MZ, and this expression is LT dependent (1). Staining with Ab's to VCAM-1 revealed intense staining within the GC/FDC network, consistent with expression of VCAM on both murine (35) and human FDCs (36). Importantly, a thin layer of VCAM-1 expression (green) could be detected proximal to the Asm-1 stain (red) (see arrows, Figure 5, c and e). In contrast, VCAM-1 expression, in conjunction with the Asm-1 stain (red), was barely detectable in the MZ from spleens of LT $\beta \mathrm{R}$ Ig-treated animals (Figure 5, d and f). In some cases, remnants of VCAM- 1 expression could be detected in
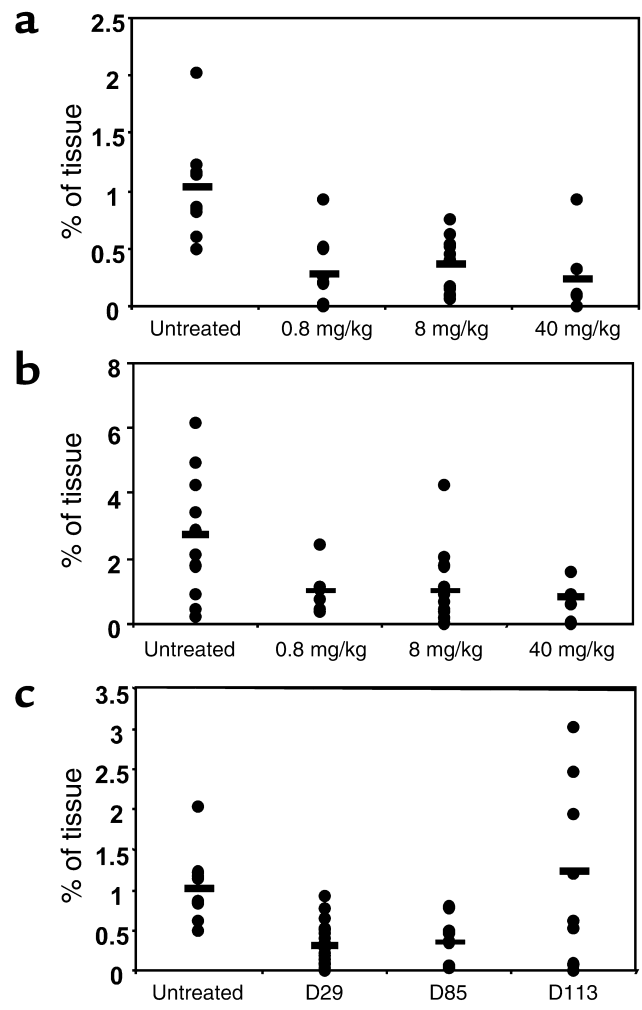

\section{Figure 4}

FDC network areas in the spleen are significantly diminished following treatment with LT $\beta$ R-Ig in all dose groups. Spleen sections stained with anti-CD35 Ab's were subjected to morphometric analysis. FDC networks in the spleen ( $\mathbf{a}$ and $\mathbf{c}$ ) and $\mathrm{LN}(\mathbf{b})$ were quantified in terms of areas compared with overall tissue area. For D29 (day 29) data, $8-\mathrm{mg} / \mathrm{kg}$ intravenous and subcutaneous groups were combined. For recovery data $(\mathbf{c})$, all doses were combined. 

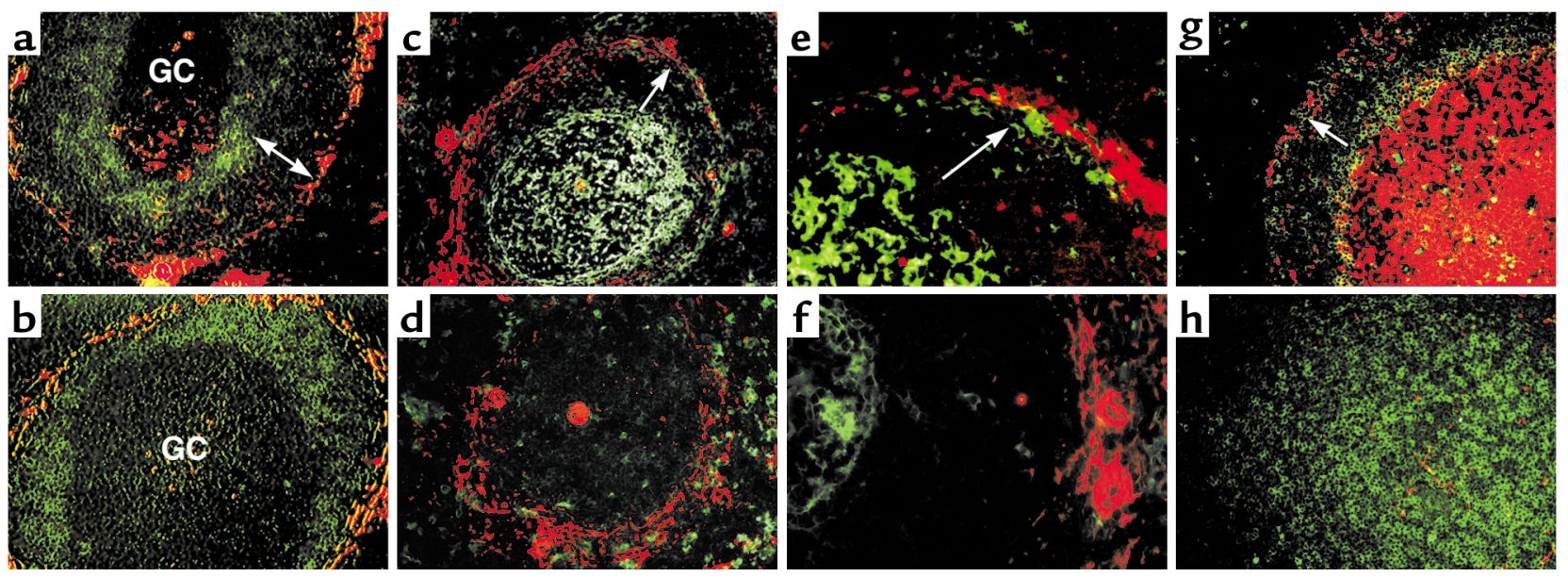

\section{Figure 5}

LT $\beta R$-Ig treatment results in changes in the splenic MZ. Spleens from untreated (a, $\mathbf{c}, \mathbf{e}, \mathbf{g})$ and treated $(8 \mathrm{mg} / \mathrm{kg} \mathrm{LT \beta R}-\mathrm{lg})$ animals $(\mathbf{b}, \mathbf{d}, \mathbf{f}, \mathbf{h})$ were stained with Asm-1 (red) and anti-IgD (green) (a, b), or stained with Asm-1 (red) and anti-VCAM-1 (green) (c-f), or stained with anti$\operatorname{lgD}$ (green) with anti-VCAM-1 (green) $(\mathbf{g}-\mathbf{h})$. The i-MZ is identified (a) with arrows. The Asm-1/VCAM-1-positive MZ is identified (c, e, $\mathbf{g})$ with arrows. Representative spleens were examined by fluorescence microscopy and were photographed at $\times 100$ (a, b, c, d, g, h) or 200×(e-f).

what looked to be decaying GC (Figure 5f). Thus, not only is FDC-associated expression of CD35 and CD21 complement receptors absent after treatment, but this correlates with other FDC markers such as VCAM-1. In agreement with these observations, detection of VCAM-1 in conjunction with IgD confirmed the MZ and GC location of VCAM-1 expression in untreated (Figure $5 \mathrm{~g}$ ), but not LT $\beta \mathrm{R}-\mathrm{Ig}$-treated, (Figure $5 \mathrm{~h}$ ) animals. It is possible that the VCAM-1-positive cells in the $\mathrm{i}-\mathrm{MZ}$ are precursor FDCs. Therefore, treatment with LT $\beta$ R-Ig affects the primate MZ microenvironment despite key architectural differences in this structure between the rodent and primate.
Immune complex deposition in primates. Because FDCs retain antigen in the form of IC (12), FDC function may be assessed by evaluating preformed IC trapping in the spleen. IC applied directly to frozen primate spleen sections in vitro are observed to localize to the FDC network in a uniform manner (red) (Figure 6, a and b). However, in agreement with IC trapping on human tissues (37), in the absence of fresh serum, in vitro trapping of IC was inhibited, suggesting that IC trapping on monkey FDCs is complement dependent (Figure 6, c and d). Interestingly, dependence on serum for IC trapping to FDC networks was not observed for in vitro IC deposition onto mouse splenic tissue (data
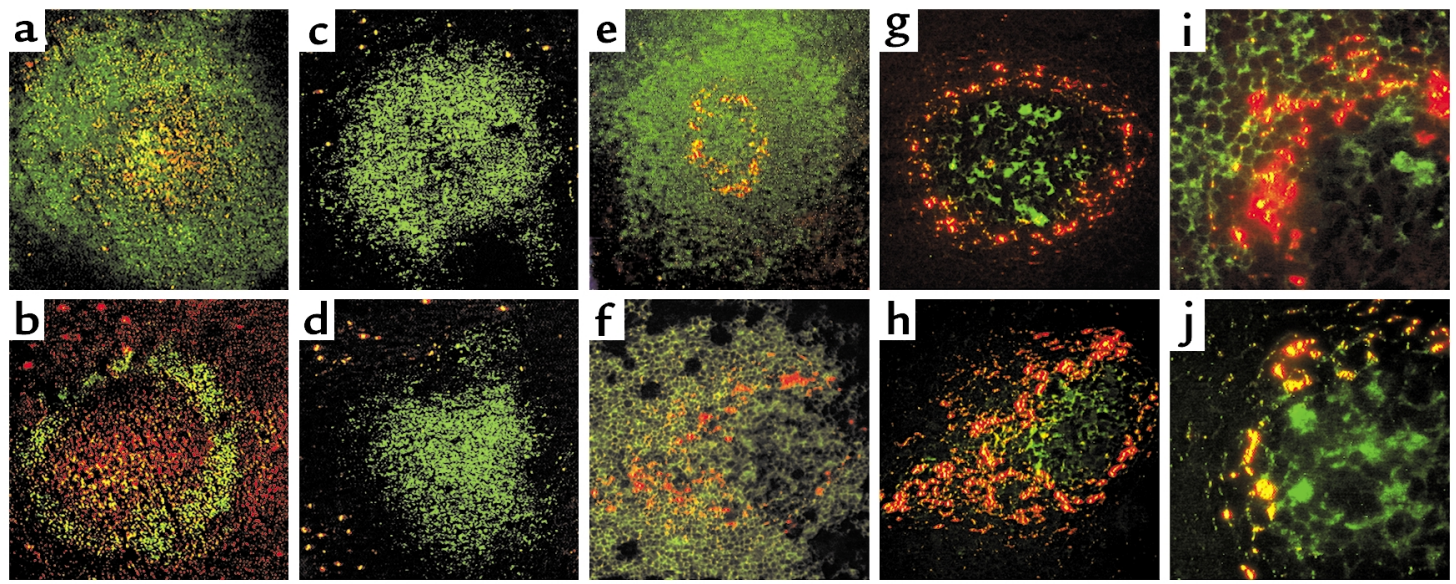

\section{Figure 6}

Immune complex trapping in primate spleens. Spleens from untreated primates were stained with PAP in vitro in the presence (a and $\mathbf{b})$ or absence ( $\mathbf{c}$ and $\mathbf{d}$ ) of $10 \%$ fresh human serum and stained with FITC anti-CD20 Ab's (green) and TSA-rhodamine (red) to develop PAP. In other studies, primates were injected with $25 \mathrm{mg} / \mathrm{kg}$ of PAP 24 hours before sacrifice. Spleen sections from these animals were stained with TSA-rhodamine (red) to develop endogenous peroxidase from the PAP and stained with FITC anti-CD20 Ab's (e, f, i) to identify mantle area and FITC anti-CD35 Ab's ( $\mathbf{g}, \mathbf{h}, \mathbf{j})$ to identify FDC networks. Representative spleens from three animals were examined by fluorescence microscopy and photographed at $\times 100(\mathbf{a}-\mathbf{h})$ or $\times 200$ (i and $\mathbf{j})$. 

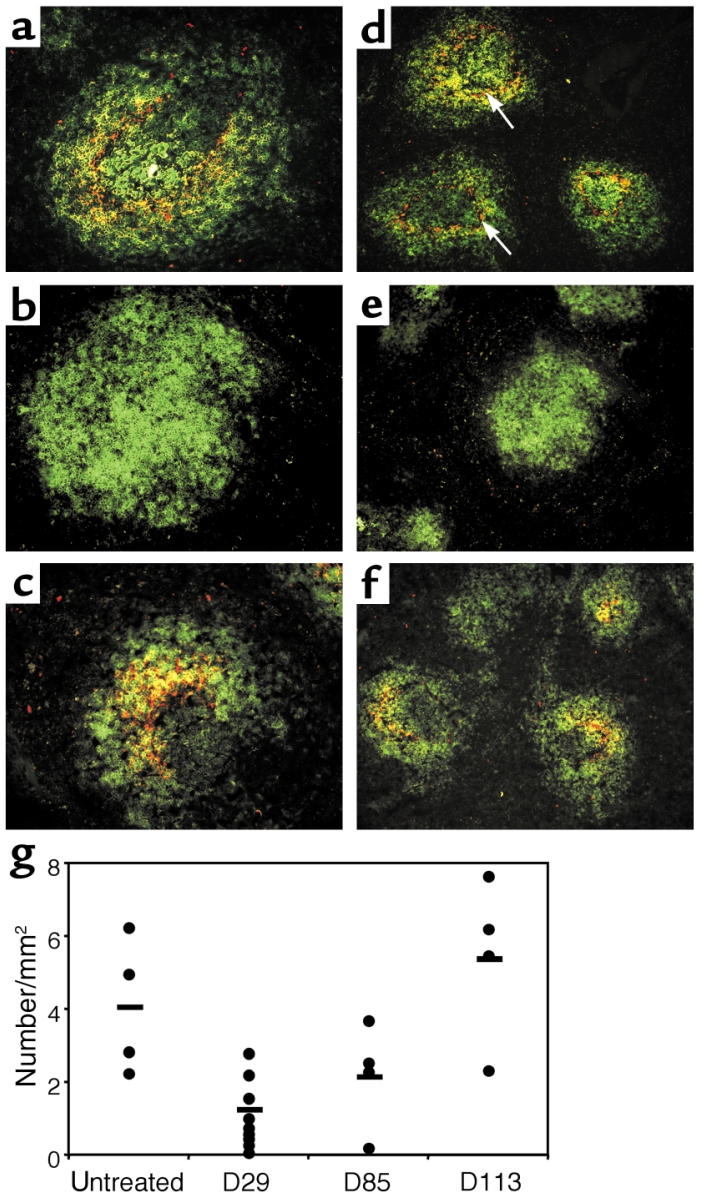

\section{Figure 7}

FDC function is impaired in animals treated with LT $\beta R$-Ig, and FDCs recover function after 3 months. Spleens from untreated ( $\mathbf{a}$ and $\mathbf{d}$ ), treated ( $8 \mathrm{mg} / \mathrm{kg} \mathrm{LT} \beta \mathrm{R}-\mathrm{lg})$ (b and $\mathbf{e})$, and recovery animals $(8 \mathrm{mg} / \mathrm{kg}$ treatment followed by 3 -month recovery) ( $\mathbf{c}$ and $\mathbf{f}$ ) injected with PAP were stained with anti-CD20 (green) to identify B cell follicles and TSA-rhodamine (red) to identify PAP networks. PAP networks are visible within CD20+ $\mathrm{B}$ cell follicles (arrows). (a, b, c) 100×. (d, e, f) $\times 50$. Spleen sections were analyzed for the number of PAP networks in a given area of tissue both after treatment and after recovery periods. Doses were combined for each time point $(\mathbf{g})$.

not shown). This suggests that these two species diverge in the requirement for complement in IC trapping and that in the mouse, $\mathrm{Fc}$ receptors may be sufficient for this process. In agreement with this hypothesis, Yoshida et al. have shown that in vitro IC trapping in mouse secondary follicle GCs could be induced in the absence of serum (38); however, IC trapping in vivo appears to be dependent on FDC-specific expression of complement receptors (39).

Using methodology developed from rodent studies, the process of IC trapping in vivo in primates was also studied by injecting $25 \mathrm{mg} / \mathrm{kg}$ IC intravenously 24 hours before sacrifice $(40,41)$. Splenic sections were then stained with anti-CD20 mAb to detect mantle and MZ B cells, or with anti-CD35 mAb to detect FDC networks (green), and costained with TSA-rhodamine to detect peroxidase from the PAP ICs (Figure 6, e-j). In most follicles, PAP-positive cells were located in a ring within the CD20-positive follicle (Figure 6, e-f) that encircled the CD35-positive FDC network (Figure $6, \mathrm{~g}$ and $\mathrm{h}$ ). Since in primates most of the follicles contain GCs, IC-injected animals exhibit spleens filled with these ringlike networks. Higher magnification reveals that these PAP-positive cells have a dendritic morphology and costained with anti-CD35 (yellow cells, Figure 6j), but not with anti-CD20 Ab's (Figure 6i), consistent with the interpretation that these are, in fact, genuine FDCs.

Delineation and quantification of functional FDC networks. Using the in vivo IC-trapping approach, FDC function was evaluated in treated and untreated primates. In control animal spleens, the ringlike PAP networks are detected in many of the $\mathrm{CD} 20^{+}$-stained $\mathrm{B}$ cell follicles (Figure 7, a and d, arrows). However, monkeys treated with as little as $0.8 \mathrm{mg} / \mathrm{kg}$ LT $\beta$ R-Ig exhibit impaired IC trapping as evidenced by the decreased numbers of PAP networks (Figure 7, b, e, and g) $(P<0.05)$. Recovery of IC trapping paralleled the restoration of CD35 expression (Figure 7, c, f, and g). Likewise, the restored PAP networks in many follicles were also broader and more diffuse compared with mature networks as was observed with CD35 recovery (see Figure 7f). IC is trapped primarily in the spleen and liver, and the LN does not have immediate access to blood-borne antigen. Antigen would need to enter via the lymph to become trapped within the LN. As expected, very few LN sections contained PAP-positive networks, and therefore they were not quantified.

Using IgD to demarcate the mantle or primary follicle from the GC and the MZ, PAP deposition was observed
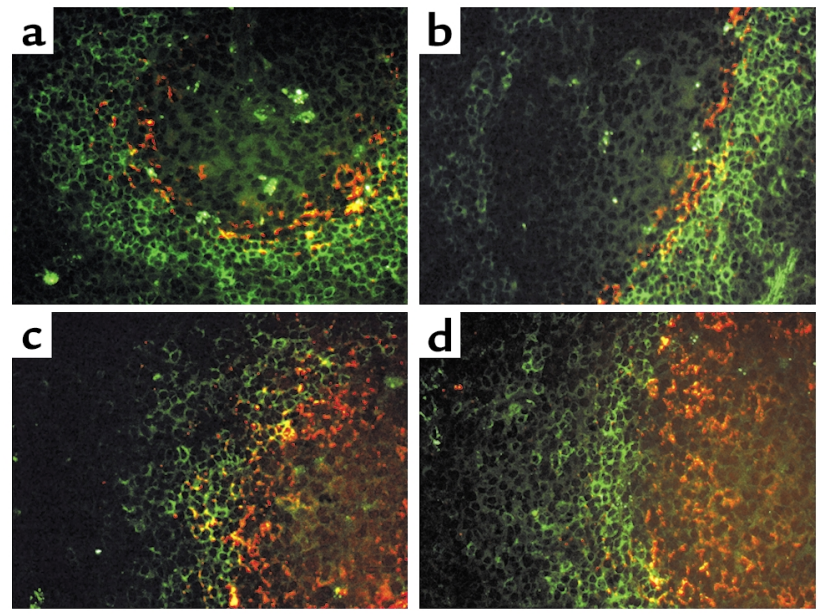

\section{Figure 8}

Location of PAP-positive cells in untreated animals compared with animals with recovery FDC networks. Spleens from untreated ( $\mathbf{a}$ and b) and recovery animals (c and d) $(8 \mathrm{mg} / \mathrm{kg}$ treatment followed by 3-month recovery) injected with PAP were stained with TSA-rhodamine (red) to visualize ICs and anti-IgD (green) to determine the location of PAP-positive cells. Representative spleens were examined by fluorescence microscopy and photographed at $\times 100$. 


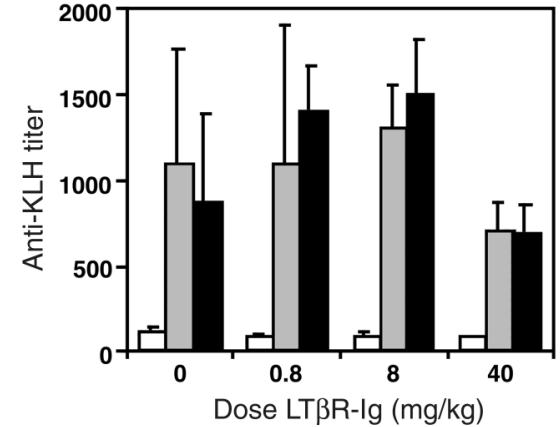

Figure 9

$\mathrm{KLH}$ Ab responses are unaffected by LT $\beta R$-Ig treatment. Serum was collected from primates immunized with KLH in IFA at day 7 (white bars), 14 (gray bars), and 20 (black bars) and subjected to ELISA to determine relative titers of anti-KLH Ab's. Titers were normalized over preimmunized anti-KLH levels for each animal, which typically ranged from 1 to 50 . The data from both $10 \mathrm{mg} / \mathrm{kg}$ intravenous and $10 \mathrm{mg} / \mathrm{kg}$ subcutaneous immunization routes were combined, and the number of animals in each group was at least ten. Error bars represent SEM.

on the rim of the GC in untreated animals, although a small amount of PAP deposition was also noted in the IgD-positive mantle (Figure 8, a and b). We cannot discount the possibility that PAP-positive cells originate elsewhere, e.g., from the MZ. We suspect that analysis at 24 hours after PAP injection prevented visualization of the earliest PAP-positive cells and, indeed, in the LN migration of antigen transport cells occurs as early as 1 minute after local HRP injection and is complete by 24 hours (42). Taken together, the ringlike appearance of IC in vivo around FDC networks indicates that IC must be deposited or transferred from transport cells that move through the mantle zone to the outer ring of the GC. In the case of animals recovering from LT $\beta$ R-Ig treatment, PAP was clearly localized to FDC-like cells in a broad diffuse band (Figure 8, $\mathrm{c}$ and d). In contrast to untreated animals, the PAP-positive cells appeared to move deeper into the IgD-negative GC region. We postulate that in an untreated animal, tightly formed FDC networks in active GCs prevent immigration of PAP carrying FDC-like cells into the GC and restrict their localization to the $\mathrm{GC}$ rim. In the case of animals recovering from treatment, the FDC network appears loosely aggregated and diffuse, suggesting that this microenvironment may not have completely reestablished chemokine gradients, thus permitting the entry of new FDC-like IC-carrying cells.

Evaluation of KLH Ab responses. Because LT $\beta$ R-Ig treatment in primates results in the loss of FDC networks and suppression of IC trapping, it was of interest to determine if treatment had an impact on a $T$ cell-dependent humoral response. In $L T \alpha^{-/}$and $L T \beta^{-/-}$ mice, initial titers of the primary $\mathrm{Ab}$ response to nitrophenyl (NP) administered in adjuvant were not significantly affected by the absence of LT pathway; however, affinity maturation was impaired (43). In $L T \beta R^{-/}$ mice, impaired affinity maturation and a reduction in
NP-specific IgG1 are only observed with low doses (5 $\mu \mathrm{g} /$ mouse) of NP (44). Similar observations have been noted in $L T \alpha^{--}$mice where high doses of antigen resulted in somatic mutations in the $V_{b} 186.2$ gene segment (45). In the adult mouse, LT $\beta R$-Ig treatment did not impact the affinity maturation of the NP response using $100 \mu \mathrm{g} /$ mouse of NP (Vora and Browning, unpublished observations). With respect to the KLH response in mice, anti-KLH titers developed slightly more slowly but reached control levels in immunized mice treated with LT $\beta R$ - $\operatorname{Ig}(4)$. However, in this study, examination of anti-KLH titers in monkeys from serum collected at days 7,14 , and 20 days after immunization revealed no significant differences between treatment and control groups and no kinetic lag was observed in the treated animals (Figure 9).

\section{Discussion}

These results show that inhibition of the LT pathway alters the FDC and MZ microenvironments in a primate. Following a 4-week treatment of LT $\beta$ R-Ig, CD21, $\mathrm{CD} 35$, and VCAM, staining diminished or disappeared. Coincident with FDC disappearance, injected immune complexes did not trap within the outer edges of the germinal centers, indicating that the FDC networks were not only invisible, but also functionally impaired. In addition, the i-MZ collapsed with treatment, resulting in the myofibroblast layer directly abutting the $\mathrm{IgD}^{+}$primary follicle, and VCAM- 1 expression in the MZ was vastly reduced. Lastly, FDC networks reappeared and regained IC trapping function following clearance of the inhibitor, although the networks appeared diffuse, and IC bearing cells distributed more loosely in and around the GC.

We had established previously in nonimmunized mice that FDC networks required continual LT $\beta$ R signaling to remain mature (6). Because of significant differences in splenic morphology and the fact that most of the white pulp contains secondary rather than primary follicles in the primate, it was unclear if the LT system would serve the same regulatory function in primates as it does in rodents. The results presented here show that even in a mixed collection of new and aging GCs, LT inhibition results in downmodulation of FDC networks. The loss of FDC networks appears to be uncoupled from the maintenance of GC integrity. IgDnegative regions corresponding to GCs can be observed that have been emptied of CD35-positive resident FDCs, yet contain Ki-67-positive cells that appear to be proliferating $\mathrm{B}$ cells. Consistent with this observation, $L T \beta^{-/-}$mice that lack FDC networks show evidence of GC-like cell clusters in B cell follicles following immunization, and FDC-less GCs can be seen in the lymph nodes of CXCL13 chemokine-deficient mice $(2,46)$.

Compared with the rodent system, the architecture of primate and human $\mathrm{MZ}$ remains poorly understood, and outside of histological observations, there are few if any functional analyses. In humans and primates, the $\mathrm{i}-\mathrm{MZ}$ is sparsely populated with $\mathrm{IgD}$-positive $\mathrm{B}$ cells as 
opposed to the mantle that is rich in follicular IgD-positive cells. Using fluorescence microscopy, changes in this microenvironment are evident with treatment. We found that the IgD-negative i-MZ collapsed with LT $\beta R$ Ig treatment, consistent with the observations that this compartment is under LT control in rodents (4). The ability to modulate the $\mathrm{i}-\mathrm{MZ}$ by LT inhibition indicates that this is a distinct compartment. We also observed that LT $\beta$ R-Ig treatment resulted in vastly reduced VCAM-1 expression in the MZ, in agreement with data in the mouse (1). What type of MZ cells display VCAM is not clear, but precursor FDC or stromal elements are possibilities. We were unable to find suitable markers for the outer MZ (o-MZ) and hence could not determine if the o-MZ was also under LT control. This question is of potential interest since the function of the o$\mathrm{MZ}$ is unknown, and, moreover, this zone abuts the perifollicular zone. The perifollicular zone contains sailoadhesin-positive macrophages that may resemble similar cells in the rodent MZ. These macrophages surround sheathed capillaries that are possibly candidates for the antigen-sensing structures corresponding to the rodent MZ sinus (34). Since primates lack a marginal sinus, it is unclear how blood-borne antigen is presented to antigen-presenting cells in the spleen (4). Exploring how primates treated with LT $\beta$ R-Ig respond to such antigens will therefore be of interest. Curiously, $\mathrm{T}$ cells are present in the $\mathrm{i}-\mathrm{MZ}$ and myofibroblast region in a manner that varied from follicle to follicle, even within one spleen (34). The rodent MZ, by comparison, is relatively free of $\mathrm{T}$ cells. This region may therefore have a function that is not exactly paralleled in the rodent. These results are the first to show that the primate $\mathrm{MZ}$ can be modulated.

This study was also designed to determine if FDC function, as measured by IC trapping, is impaired with LT $\beta$ R-Ig treatment. By injecting preformed IC 24 hours prior to sacrifice, we have visualized IC-bearing FDClike cells arranged in a ringlike network that localizes to the rim of the GC. Moreover, these networks are significantly decreased in treated animals and recover after 3 months. Hence, LT $\beta$ R-Ig treatment not only dissolves FDC networks but also prevents further IC trapping in lymphoid tissues.

The images of IC trapping onto the FDC network reported here are consistent with what is known about antigen transport to the FDC network in the rodent (42, $47,48)$. In the spleen, antigen is presumably captured by cellular elements that sample blood percolating through the MZ. In the lymph node, cell bound and/or soluble antigen enters with the lymph and localizes to the subcapsular sinus. Antigen is either transferred directly to precursors of the FDC or via ill-defined antigen-transporting cells that pass the antigen to the FDC networks $(42,49)$. While this analysis was confined to a relatively late time point, these data show that essentially all of the IC-positive cells at 24 hours are FDC-like, i.e., CD35 positive and CD20 negative. Moreover, we have observed in some samples a continuum of PAP staining that extends through the mantle zone and forms a ring around preexisting GCs. This observation suggests the movement of IC-positive FDC-like cells toward the GC. In this case, FDCs would appear to be quite mobile, which is perhaps consistent with their surprisingly fast disappearance following $\mathrm{LT}$ inhibition in the rodent. In animals that were allowed to recover from LT $\beta R$-Ig treatment, the IC-positive FDC-like cells were also located near the GC; however, in some cases, they were found within the GC itself and not restricted to the rim of the GC. This may reflect the relatively loose, diffuse structure of the recovering GC. In most cases where in vivo IC deposition has been examined in the rodent, these ringlike structures have not been observed (4), and hints of a ring conformation were only revealed in $F c R \gamma$ chain knockout animals where IC deposition was enhanced (41). It is possible that IC deposition may be somewhat different in clean-caged mice that have been immunized against only a small number of antigens, as opposed to primates that encounter more frequent and diverse immunological challenges.

After a recovery phase, CD35-positive, IC-positive, FDC-like networks often reappear as larger, more diffuse structures when compared with the mature dense networks in untreated animals. While we view these diffuse structures as nascent networks, the exact interpretation is complicated by our inability to differentiate between primary follicles, vestigal decaying GCs, viable FDC-less GCs, and de novo GC events. FDC network recovery was evident in the spleen, but the networks in the $\mathrm{LN}$ did not recover to a normal level, indicating a much slower process. There are hints that FDC networks in the LN may differ from those in the spleen, but the details remain obscure (2). The observations in the spleen indicate that the microenvironmental changes are reversible, which is consistent with the relatively plastic nature of the immune organs.

The effects of LT $\beta$ R signaling on FDC function probably occur at multiple levels. First, LT signaling is likely required for the formation of FDC precursors since most samples from treated animals exhibited a fairly complete absence of PAP deposition. Secondly, the maintenance of mature FDCs requires continual LT signaling as evidenced by the complete absence of FDCs within secondary follicles in treated animals. Finally, while not yet formally demonstrated, it is possible that cells in the GC produce a chemokine that forces FDC migration from the mantle into the GC, and this process may be under LT/TNF control. The diffuse pattern of CD35 staining observed during recovery from LT $\beta$ R-Ig treatment suggests that during the early stages of recovery, the chemokine gradient has not yet reestablished itself, and hence the FDC have not completely migrated and compacted into the GC.

Here, we found that LT $\beta$ R-Ig treatment had no effect on the response to a neo-antigen, $\mathrm{KLH}$, and this result is consistent with observations in mouse experiments, although different adjuvants were used, i.e., IFA versus alum (4). If FDCs are the major cell type required for 
antigen trapping, eliminating this antigen reservoir should perhaps lead to compromised affinity maturation of the humoral response. Evaluation of the quality of $\mathrm{Ab}$ responses typically has been difficult in the rodent, with the NP system being one of the most intensely studied. In the mouse, the linkage between FDC networks and affinity maturation has remained poorly resolved for several reasons. First, LT-deficient mice have been used to assess affinity maturation with varying results; however, these mice lack LNs, thus complicating analyses. In addition, other confounding factors include the amount of antigen, the adjuvant, and route of administration (4, 43-45, 50). Using LT $\beta R$-Ig treatment to eradicate FDC networks in normal mice, we did not observe impaired affinity maturation in the standard NP-KLH system, although a relatively large antigen dose was used (Browning and Vora, unpublished observations). Assessment of the impact of LT $\beta$ R-Ig treatment on affinity maturation in the primate will probably require further refinement of the available indicator systems in the rodent.

There are several reasons why the use of LT $\beta$ R-Ig to disrupt FDCs and the splenic MZ may have important therapeutic consequences. First, FDCs are an important reservoir for some pathogens such as HIV and $\mathrm{PrPsc}$, thus removal of this reservoir may represent a novel therapy for dealing with these infectious particles. Second, FDCs may promote survival of LN-residing lymphomas, and FDC-like cells appear in the synovial microenvironment in some rheumatoid arthritis patients; hence, elimination of FDCs could potentially cut off inappropriate survival signals in pathogenic settings. Finally, $\mathrm{CD} 27^{+}$memory B cells are found in the splenic MZ in humans (51), and cells within this microenvironment may be important in systemic humoral-based autoimmune diseases-hence disruption of the MZ microenvironment may also have a therapeutic benefit. Therefore, despite several differences between rodent and primate splenic architecture, this study demonstrates that FDC networks and the splenic MZ in these species are controlled by the LT system in a similar manner. Moreover, the differences in primate architecture, in particular the predominance of GCs of various stages throughout the spleen, have been exploited in this study to assess FDC migration, recovery, and function, as well as an evaluation of LT-sensitive aspects of the MZ. Given its ability to selectively manipulate elements of the immunological microenvironment in primates, LT $\beta$ R-Ig represents a novel approach for the treatment of human disease.

\section{Acknowledgments}

We wish to thank Joseph Amatucci, Konrad Miatkowski, David Griffiths, Kalpit Vora, Gerry Majeau, Paula Hochman, Tom Crowell, Joan Lane, and Christine Chutkowski at Biogen Inc., and Curtis Chan at Sierra Pathology Services for various contributions. We also thank Jason Cyster from the University of California
San Francisco for helpful information. Special thanks to Evangelia Notidis and Tony de Fougerolles for critical reading of this manuscript.

1. Lu, T.T., and Cyster, J.G. 2002. Integrin-mediated long-term B cell retention in the splenic marginal zone. Science. 297:409-412.

2. Ansel, K.M., et al. 2000. A chemokine-driven positive feedback loop organizes lymphoid follicles. Nature. 406:309-314.

3. Ware, C.F., VanArsdale, T.L., Crowe, P.D., and Browning, J.L. 1995. The ligands and receptors of the lymphotoxin system. Curr. Top. Microbiol. Immunol. 198:175-218.

4. Mackay, F., et al. 1997. Lymphotoxin but not tumor necrosis factor functions to maintain splenic architecture and humoral responsiveness in adult mice. Eur. J. Immunol. 27:2033-2042.

5. Ngo, V.N., et al. 1999. Lymphotoxin alpha/beta and tumor necrosis factor are required for stromal cell expression of homing chemokines in B and T cell areas of the spleen. J. Exp. Med. 189:403-412.

6. Mackay, F., and Browning, J.L. 1998. Turning off follicular dendritic cells. Nature. 395:26-27.

7. Grimaldi, C.M., Michael, D.J., and Diamond, B. 2001. Cutting edge: expansion and activation of a population of autoreactive marginal zone $\mathrm{B}$ cells in a model of estrogen-induced lupus. J. Immunol. 167:1886-1890.

8. Groom, J., et al. 2002. Association of Baff/BLyS overexpression and altered B cell differentiation with Sjögren's syndrome. J. Clin. Invest. 109:59-68. doi:10.1172/JCI200214121.

9. Imai, Y., and Yamakawa, M. 1996. Morphology, function and pathology of follicular dendritic cells. Pathol. Int. 46:807-833.

10. Luther, S.A., et al. 2000. BLC expression in pancreatic islets causes B cell recruitment and lymphotoxin-dependent lymphoid neogenesis. Immunity. 12:471-481.

11. Ruddle, N.H. 1999. Lymphoid neo-organogenesis: lymphotoxin's role in inflammation and development. Immunol. Res. 19:119-125.

12. Van-den-Berg, T.K., Yoshida, K., and Dijkstra, C.D. 1995. Mechanism of immune complex trapping by follicular dendritic cells. Curr. Top. Microbiol. Immunol. 201:49-67.

13. Terashima, K., Dobashi, M., Maeda, K., and Imai, Y. 1992. Follicular dendritic cell and ICCOSOMES in germinal center reactions. Sem. Immunol. 4:267-274.

14. Tew, J.G., and Mandel, T.E. 1979. Prolonged antigen half-life in the lymphoid follicles of specifically immunized mice. Immunology. 37:69-76.

15. Endres, R., et al. 1999. Mature follicular dendritic cell networks depend on expression of lymphotoxin beta receptor by radioresistant stromal cells and of lymphotoxin beta and tumor necrosis factor by B cells. J. Exp. Med. 189:159-168.

16. Fu, Y.X., and Chaplin. D.D. 1999. Development and maturation of secondary lymphoid tissues. Annu. Rev. Immunol. 17:399-433.

17. Kapasi, Z.F., et al. 1998. Follicular dendritic cell (FDC) precursors in primary lymphoid tissues. J. Immunol. 160:1078-1084.

18. Kagami, Y., et al. 2001. Establishment of a follicular lymphoma cell line (FLK-1) dependent on follicular dendritic cell-like cell line HK. Lenkemia. 15:148-156.

19. Spiegel, H., et al. 1992. Follicular dendritic cells are a major reservoir for human immunodeficiency virus type 1 in lymphoid tissues facilitating infection of CD4+ T-helper cells. Am. J. Pathol. 140:15-22.

20. Tenner-Racz, K., and Racz, P. 1995. Follicular dendritic cells initiate and maintain infection of the germinal centers by human immunodeficiency virus. Curr. Top. Microbiol. Immunol. 201:141-159.

21. Smith, B.A., et al. 2001. Persistence of infectious HIV on follicular dendritic cells. J. Immunol. 166:690-696.

22. Brown, K.L., et al. 1999. Scrapie replication in lymphoid tissues depends on prion protein-expressing follicular dendritic cells. Nat. Med. 5:1308-1312.

23. Mabbott, N.A., Mackay, F., Minns, F., and Bruce, M.E. 2000. Temporary inactivation of follicular dendritic cells delays neuroinvasion of scrapie. Nat. Med. 6:719-720.

24. Lindhout, E., et al. 1999. Fibroblast-like synoviocytes from rheumatoid arthritis patients have intrinsic properties of follicular dendritic cells. J. Immunol. 162:5949-5956.

25. Browning, J.L., et al. 1995. Characterization of surface lymphotoxin forms. Use of specific monoclonal antibodies and soluble receptors. J. Immunol. 154:33-46.

26. Cornacoff, J.B., et al. 1983. Primate erythrocyte-immune complex-clearing mechanism. J. Clin. Invest. 71:236-247.

27. Davies, K.A., et al. 1990. A study of in vivo immune complex formation and clearance in man. J. Immunol. 144:4613-4620.

28. Zannini, C., et al. 2000. A video densitometric analysis of viral burden and follicular dendritic cell damage in lymph nodes in the latency phase of HIV infection. Cytometry. 40:209-213.

29. Zhang, Z.Q., et al. 1999. Reversibility of the pathological changes in the 
follicular dendritic cell network with treatment of HIV-1 infection. Proc. Natl. Acad. Sci. USA. 96:5169-5172.

30. Liu, Y.J., et al. 1997. Follicular dendritic cells specifically express the long CR2/CD21 isoform. J. Exp. Med. 185:165-170.

31. Cattoretti, G., et al. 1992. Monoclonal antibodies against recombinant parts of the Ki-67 antigen (MIB 1 and MIB 3) detect proliferating cells in microwave-processed formalin-fixed paraffin sections. J. Pathol. 168:357-363.

32. Fu, Y.X., et al. 1997. Independent signals regulate development of primary and secondary follicle structure in spleen and mesenteric lymph node. Proc. Natl. Acad. Sci. USA. 94:5739-5743.

33. Gonzalez, M., et al. 1998. The sequential role of lymphotoxin and B cells in the development of splenic follicles. J. Exp. Med. 187:997-1007.

34. Steiniger, B., and Barth, P. 1999. Microanatomy and function of the spleen. Springer-Verlag. Berlin, Germany. 101 pp.

35. Koopman, G., et al. 1991. Adhesion of human B cells to follicular dendritic cells involves both the lymphocyte function-associated antigen/intercellular adhesion molecule 1 and very late antigen 4/vascular cell adhesion molecule 1 pathways. J. Exp. Med. 173:1297-1304.

36. Freedman, A.S., et al. 1990. Adhesion of human B cells to germinal centers in vitro involves VLA-4 and INCAM-110. Science. 249:1030-1033.

37. Maeda, K., et al. 1995. In vitro immune complex binding assay to examine the mechanism of immune complex trapping by human follicular dendritic cells (FDC). Dendritic Cells in Fundamental and Clinical Immunology. 2:317-319.

38. Yoshida, K., van den Berg, T.K., and Dijkstra, C.D. 1993. Two different mechanisms of immune complex trapping in the mouse spleen during immune responses. Dendritic cells in fundamental and clinical immunology. Plenum Press. New York, New York, USA. 377-381.

39. Fang, Y., et al. 1998. Expression of complement receptors 1 and 2 on follicular dendritic cells is necessary for the generation of a strong antigenspecific IgG response. J. Immunol. 160:5273-5279.

40. Van den Berg, T.K., et al. 1992. Selective inhibition of immune complex trapping by follicular dendritic cells with monoclonal antibodies against rat C3. Eur. J. Immunol. 22:957-962.
41. Vora, K.A., Ravetch, J.V., and Manser, T. 1997. Amplified follicular immune complex deposition in mice lacking the Fc receptor gammachain does not alter maturation of the B cell response. J. Immunol. 159:2116-2124.

42. Szakal, A.K., Holmes, K.L., and Tew, J.G. 1983. Transport of immune complexes from the subcapsular sinus to lymph node follicles on the surface of nonphagocytic cells, including cells with dendritic morphology. J. Immunol. 131:1714-1727.

43. Koni, P.A., et al. 1997. Distinct roles in lymphoid organogenesis for lymphotoxins alpha and beta revealed in lymphotoxin beta-deficient mice. Immunity. 6:491-500.

44. Futterer, A., et al. 1998.The lymphotoxin beta receptor controls organogenesis and affinity maturation in peripheral lymphoid tissues. Immunity. 9:59-70.

45. Matsumoto, M., et al. 1996. Affinity maturation without germinal centres in lymphotoxin-alpha-deficient mice. Nature. 382:462-466.

46. Koni, P.A., and Flavell, R.A. 1999. Lymph node germinal centers form in the absence of follicular dendritic cell networks. J. Exp. Med. 189:855-864.

47. Berney, C., et al. 1999. A member of the dendritic cell family that enters $\mathrm{B}$ cell follicles and stimulates primary antibody responses identified by a mannose receptor fusion protein. J. Exp. Med. 190:851-860.

48. Szakal, A.K., Kosco, M.H., and Tew, J.G. 1988. A novel in vivo follicular dendritic cell-dependent iccosome-mediated mechanism for delivery of antigen to antigen-processing cells. J. Immunol. 140:341-353.

49. Martinez-Pomares, L., et al. 1996. Fc chimeric protein containing the cysteine-rich domain of the murine mannose receptor binds to macrophages from splenic marginal zone and lymph node subcapsular sinus and to germinal centers. J. Exp. Med. 184:1927-1937.

50. Rennert, P.D., et al. 2001. Essential role of lymph nodes in contact hypersensitivity revealed in lymphotoxin-alpha-deficient mice. J. Exp. Med. 193:1227-1238.

51. Tangye, S.G., et al. 1998. Identification of functional human splenic memory B cells by expression of CD148 and CD27. J. Exp. Med. 188:1691-1703. 